\title{
« L’espèce de savon fait disparaître la petite main »
}

\author{
Cristina Regazzi
}

L es personnes âgées gardent les petits-enfants, sont des mamies ou des papis, s'asseyent sur des bancs et regardent la vie passer, font des gâteaux, vont se promener avec les chiens ou font de la randonnée. Contrairement aux autres citadins, ils se promènent tranquillement parmi les « travailleurs » stressés, occupent les places assises dans les trains et les trams pendant l'heure de pointe et comptent leur monnaie à la caisse des supermarchés juste avant la fermeture. Ce sont là de nombreux clichés tenaces sur les personnes âgées.

Cependant elles sont aujourd'hui plus que jamais non seulement en forme physiquement et mentalement, mais également souvent ouvertes aux nouvelles évolutions et ceci, contrairement à des préjugés discordants, face aux technologies à l'évolution fulgurante qui enrichissent (le plus souvent) notre vie.

Smartphone, tablette et ordinateur enregistrent d'importants chiffres de vente sans cesse croissants dans la tranche d'âge des plus de 65 ans. En conséquence, les personnes âgées surfent sur Internet, utilisent Skype ainsi que la fonction tactile. Déjà quand on achète une machine à laver aujourd'hui, on doit bien s'y connaître en numérique : soit on arrive à utiliser de manière intuitive le menu d'exploitation soit on se débat avec un épais manuel de l'utilisateur. Même le nouveau cuiseur vapeur dans la cuisine propose de superbes recettes rien qu'en appuyant sur un bouton, à condition de savoir comment marche ce machin. Et que dire des tableaux de bord de nos voitures qui ressemblent plus à des cockpits d'avion ou à des surfaces de jeux vidéo!

Les technologies qui nous assistent dans notre vie quotidienne, les technologies «Ambient/Active Assisted Living $(A A L)$ », sont de plus en plus présentes et de plus en plus avantageuses. Elles visent à favoriser l'autonomie et la vie à la maison pour les personnes âgées et, si l'on en croit l'industrie ou l'expérience japonaise, les robots prendront de plus en plus pied dans le domaine des soins à l'avenir.

Pas étonnant que le nombre d'offres de cours pour seniors augmente également dans le domaine des technologies de l'information. Même ma maman (80 ans), après avoir longuement hésité, a participé à un cours d'informatique il y a de cela quelques années : d'abord avec un grand respect, puis avec un grand plaisir. Elle déclenchait les rires lorsqu'elle s'énervait et criait parfois : «Aaaah!» «Si je bouge l'espèce de savon là, la petite main disparaît! » (elle a secoué la souris et a perdu de vue le curseur sur l'écran). Aujourd'hui, elle cherche bien entendu des informations et des recettes en ligne, utilise Google pour les destinations de vacances de sa fille et envoie des messages avec ou sans photo de son téléphone portable. Son modèle actuel d'ordinateur, elle l'a acheté il y a un an et tenait particulièrement à un design stylé !

Technologie et vieillesse ? Naturellement ! Mais les besoins des personnes vieillissantes doivent être pris en compte intelligemment lors de la conception et du design des produits et des prestations. Car cette clientèle (de plus en plus exigeante) a des besoins qui ressemblent souvent à ceux des personnes plus jeunes car ces derniers également ne souhaitent pas lire le manuel de 250 pages de la nouvelle machine à laver !

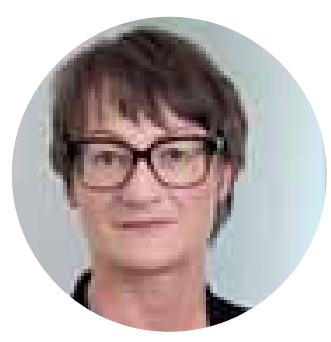

Cristina Regazzi Gérontologue Hochschule Luzern (HSLU), directrice centre pour personnes agées (Richterswil/Samstagern), experte en marketing/communication

c.regazzi@bluewin.ch 